\title{
Preditores da Adição ao Trabalho em Trabalhadores que utilizam Tecnologias de Informação e Comunicação
}

\author{
Mary Sandra Carlotto ${ }^{1}$ \\ Programa de Pós-Graduação em Psicologia da Pontifícia Universidade Católica \\ do Rio Grande do Sul, Porto Alegre, Rio Grande do Sul, Brasil \\ Guilherme Welter Wendt \\ Unit for School and Family Studies of the Goldsmiths College at University of London, \\ London, Grã-Bretanha \\ Carolina Lisboa \\ Programa de Pós-Graduação em Psicologia da Pontifícia Universidade Católica \\ do Rio Grande do Sul, Porto Alegre, Rio Grande do Sul, Brasil \\ Marcela Alves de Moraes \\ Programa de Pós-Graduação em Psicologia da Pontificia Universidade Católica \\ do Rio Grande do Sul, Porto Alegre, Rio Grande do Sul, Brasil \\ Bolsista de Iniciação Científica PBIT/Cnpq
}

\begin{abstract}
Resumo
Denomina-se por adição ao trabalho a combinação de trabalho excessivo e necessidade de trabalhar constantemente o que pode desencadear problemas físicos e psicossomáticos. Este tema tem recebido especial atenção da comunidade científica uma vez que, em casos de adição ao trabalho, o indivíduo vai perdendo gradativamente o controle emocional em relação às exigências ocupacionais. Assim, o presente estudo buscou verificar se variáveis sociodemográficas, laborais, tecnoestresse e satisfação com a vida predizem adição ao trabalho em trabalhadores que utilizam Tecnologias de Informação e Comunicação (TICs) nas suas atividades laborais. Trata-se de um estudo de delineamento observacional analítico transversal, sendo a amostra composta por 88 trabalhadores que utilizam em seu trabalho TICs. Os resultados do estudo revelam que as dimensões do tecnoestresse explicam a adição ao trabalho, corroborando modelos teóricos que apontam para uma associação entre utilizar excessivamente tecnologia e maior tendência para desenvolver adição ao trabalho. A dimensão ansiedade, quanto ao uso de TICs, revelou maior poder explicativo para as dimensões da adição ao trabalho: trabalho excessivo e trabalho compulsivo. No tocante à dimensão trabalho excessivo, verificou-se ainda que a descrença sobre o uso de TICs explica, juntamente com a ansiedade, comportamentos de trabalhar excessivamente. As implicações destes resultados podem ter especial importância na implementação e reorganização de rotinas de trabalho, nas políticas de saúde do trabalhador assim como no campo da prevenção e intervenção psicossocial e clínica.
\end{abstract}

Palavras-chave: Adição ao trabalho, preditores, tecnologias de informação e comunicação.

Endereço para correspondência: Programa de Pós-Graduação em Psicologia, Pontifícia Universidade Católica do Rio Grande do Sul, Av. Ipiranga, 6681, Partenon, Porto Alegre, RS, Brasil 90619-900. E-mail: mscarlotto@ gmail.com, guilhermewwendt@gmail.com, lisboacaro@gmail.com e marcela.alves.moraes@gmail.com Apoio: Coordenação de Aperfeiçoamento de Pessoal de Nível Superior (CAPES, processo BEX 893713-3). 


\title{
Predictors of Work Addiction in Workers who Use Information and Communication Technologies
}

\begin{abstract}
Workaholism is defined by the combination of overwork and the need to constantly work, which can result in individual's physical and psychosomatic problems. This topic has been receiving special attention from the scientific community, since workaholism could gradually make individuals lose emotional control over their occupational demands. Thus, the present study aimed to verify whether sociodemographic and labor characteristics, technostress and life satisfaction predict workaholism in professionals who use Information and Communication Technologies (ICT) at their work. This is an observational analytical cross-sectional study, and the sample is constituted by 88 participants who use ICT. The results showed that the dimensions of technostress explain workaholism, corroborating theoretical models that show an association between the overuse of technology and a higher tendency to develop workaholism. The dimension anxiety related to the use of ICT showed higher explanatory power for the dimensions of workaholism: excessive work and compulsive work. Regarding the dimension excessive work, it was found that disbelief about the use of ICT explains, along with anxiety, the behavior of overwork. These findings' implications may have particular importance in the implementation and reorganization of work routines, workers' health policies, as well as in the field of prevention, and clinical and psychosocial intervention.
\end{abstract}

Keywords: Workaholism, predictors, information and communication technologies.

\section{Predictores de Adición al Trabajo em los Trabajadores que Utilizan las Tecnologías de Información y Comunicación}

\section{Resumen}

Definiese por adicción al trabajo la combinación de trabajo excesivo y la necesidad de trabajar constantemente lo que puede llevar a problemas físicos y psicosomáticos a lo individuo. Este tema ha sido objeto de especial atención por parte de la comunidad científica, ya que en la adicción al trabajo, poco a poco, el individuo pierde el control emocional sobre las demandas laborales. Por lo tanto, el presente estudio tuvo como objetivo verificar si las características sociodemográficas y laborales, el tecnoestrés y la satisfacción con la vida predicen la adicción al trabajo en trabajadores que utilizan las Tecnologías de la Información y Comunicación (TIC's) en su trabajo. Se trata de un estudio con diseño analítico observacional transversal y la muestra fue de 88 participantes que utilizan TIC's. Los resultados mostraron que las dimensiones del tecnoestrés explican la adicción al trabajo, corroborando los modelos teóricos que también muestran una asociación entre el uso excesivo de la tecnología y una mayor tendencia a desarrollar la adicción al trabajo. La dimensión ansiedad relacionada con el uso de las TIC's mostró mayor potencia explicativa para las dimensiones de la adicción al trabajo: trabajo excesivo y trabajo compulsivo. Referente a la dimensión de trabajo excesivo, se encontró que la incredulidad sobre el uso de TIC's explica, junto con la ansiedad, los comportamientos de trabajar en exceso. Las implicaciones de estos hallazgos pueden tener especial importancia en la ejecución y la reorganización de las rutinas de trabajo, en las políticas de salud de los trabajadores, así como también para el campo de la prevención y la intervención clínica y psicosocial.

Palabras clave: Adición al trabajo, preditores, tecnologías de la información y comunicación. 
A adição ao trabalho é caracterizada pelo trabalho excessivo associado a uma necessidade de trabalhar constantemente (Salanova, Del Libano, Llorens, Schaufeli, \& Fidalgo, 2008). Sendo entendida como uma patologia que se desenvolve de maneira processual, a adição ao trabalho faz com que, gradativamente, o indivíduo perca o controle emocional sobre suas atitudes em função de uma necessidade intensa de realizar uma grande quantidade e obter sucesso em suas tarefas laborais (Killinger, 1991). Além disso, pessoas adictas ao trabalho em geral podem ter dificuldades nas relações interpessoais com colegas, assim como referirem sentimentos de exaustão física e estresse mental (Wijhe, Peeters, \& Schaufeli, 2013).

Nesta interação dinâmica, crenças irracionais são constituídas e reforçam os comportamentos, processo mediado pela emoção. Estas crenças estão relacionadas à onipotência (Só eu consigo fazer este trabalho), a dificuldades em delegar (Se eu não fizer, ninguém fará) e a catastrofizações (Se eu não realizar esta tarefa ou concluir esta atividade, uma tragédia acontecerá) e são consideradas distorções cognitivas (Beck, 1997) identificadas em trabalhadores que apresentam adição ao trabalho (Wijhe et al., 2013). Assim, nesta busca incessante em superar as próprias expectativas, a pessoa que apresenta adição ao trabalho pode extrapolar os limites ou demandas exigidos pela organização, dessa maneira, portando-se de modo desadaptativo diante das tarefas e exigências laborais (Moreno-Jiménez, Gálvez-Herrer, Garrosa-Hernández, \& Rodríguez Carvajal, 2005). Como critérios para identificação ou diagnóstico desta patologia laboral, está o comportamento não só de exercer atividades laborais em alta quantidade, mas, principalmente, de trabalhar além do que lhe é demandado, inclusive em situações nas quais a pessoa não se encontra no contexto de trabalho, não conseguindo ficar sem trabalhar (Baruch, 2011; Serva \& Ferreira, 2006). E como se trata de comportamentos aditivos sustentados por crenças distorcidas, esta elevada quantidade de trabalho, que poderia resultar em alta produtividade para a organização, vem acompanhada de altos níveis de ansiedade, comprometendo a qualidade do trabalho e das relações interpessoais no ambiente laboral. A adição ao trabalho, geralmente, afeta as dimensões cognitiva, afetiva, comportamental e psicossocial dos indivíduos. Este fenômeno influencia na capacidade de tomada de decisão do indivíduo, interferindo negativamente nos seus comportamentos e relacionamentos de uma maneira geral e, ocasionando, ao longo do tempo, problemas no desempenho no trabalho (Fatayer, 2008). O contrário também pode ser uma possibilidade, uma vez que vulnerabilidades psicossociais e emocionais, como baixos níveis de satisfação com a vida associam-se à adição ao trabalho (Bonebright, Clay, \& Ankenmann, 2000; Salanova et al., 2008).

Convém salientar que o contexto socioeconômico atual é pautado pelas Tecnologias de Informação e Comunicação (TICs), presentes em praticamente todos os segmentos deste cenário (Salanova et al., 2008; Taris \& Schaufeli, 2007). O desenvolvimento de novas ferramentas tecnológicas para o aprimoramento das atividades laborais traz benefícios para o ambiente de trabalho, como maior agilidade e precisão, dentre outros muitos que se poderia listar. No entanto, tais ferramentas podem representar elevados custos humanos, como a necessidade constante de atualização pessoal, pressão por prazos, habilidade para o desenvolvimento de multitarefas, entre outros. Ademais, as TICs estendem o tradicional contexto de trabalho, desse modo, tornando possível que o colaborador execute de sua própria residência tarefas relativas ao seu trabalho, aumentando o número de horas dedicadas às crescentes demandas laborais (Pocinho $\&$ Garcia, 2008). Conforme os últimos dados divulgados pelo Instituto Brasileiro de Geografia e Estatística (IBGE, 2013), entre os anos de 2003 a 2006 , houve um aumento de $40,7 \%$ no número de colaboradores que atuam no segmento das TICs, compondo por volta de 3\% do universo de instituições empresariais no país, nas áreas como indústria, comércio e serviços.

Por exigir mais em termos cognitivos, requerendo maior capacidade de atenção difusa e concentrada, assim como múltiplas habilidades para solução de problemas, o trabalho com 
TICs pode acarretar em sobrecarga nos processos mentais dos trabalhadores. Esta sobrecarga, resultado da interação entre estímulos pessoais internos e interpretação distorcida da realidade (necessidades e perspectivas) com estímulos externos (pressão social, demandas do trabalho), torna-se cada vez mais recorrente (Salanova, Llorens, \& Cifre, 2013). Este processo pode resultar em tecnoestresse que é um estado psicológico negativo de exaustão emocional relacionado diretamente com o uso de TICs no ambiente de trabalho ou à ameaça de seu uso no futuro. O tecnoestresse é constituído por quatro dimensões: descrença, ansiedade, fadiga e ineficácia (Salanova, Llorens, Cifre, \& Nogareda, 2007). Como referido anteriormente, portanto, um grupo de profissionais que, a princípio, pode estar especialmente vulnerável a sofrer com este tipo de estresse são aqueles que atuam diretamente com TICs no seu trabalho. Diante das características específicas das demandas deste tipo de trabalho, presume-se que o tecnoestresse possa ser um fenômeno frequente, gerando desequilíbrios psicossomáticos e doenças ocupacionais (Ferreira, 2006; Pacheco, Ferreira, Pereira, \& Pereira, 2005), sendo a adição ao trabalho uma destas (Schiffrin, Edelman, Falkenster, \& Steward, 2010). Assim sendo, pode-se concluir que tecnoestresse e adição ao trabalho são fenômenos sobrepostos. Estimativas apontam que três, em cada dez trabalhadores, podem apresentar adição ao trabalho (Elowe, 2010), fato este que impacta na qualidade e satisfação de vida destes trabalhadores, em sua saúde física e nos relacionamentos interpessoais (Salanova et al., 2008).

Embora a adição ao trabalho e sua relação com o uso de tecnologias e tecnoestresse seja um tema já explorado na literatura internacional (Arla, Paquet, Scott, \& Hambley, 2012; Porter \& Kakabadse, 2006; Salanova et al., 2013), no Brasil, não se identificam nas principais bases de dados (i.e. Biblioteca Virtual em Saúde, Pepsic, Pubmed e Scielo) estudos que estabeleçam esta relação. Pelo exposto, o presente estudo teve como objetivo verificar se as variáveis sociodemográficas, laborais, tecnoestresse e satisfação com a vida predizem a adição ao trabalho em trabalhadores que utilizam TICs nas suas atividades laborais. Considerou a hipótese de que as variáveis sociodemográficas, laborais, tecnoestresse e satisfação com a vida funcionariam como preditoras da adição ao trabalho.

\section{Método}

\section{Delineamento e Participantes}

Trata-se de um estudo de delineamento observacional analítico transversal que utilizou uma amostra selecionada por conveniência constituída de 88 trabalhadores. Esses eram, em sua maioria, mulheres $(62,1 \%)$, solteiros $(55,7 \%)$, sem filhos $(63,5 \%)$, possuem curso superior $(96,6 \%)$ e apresentavam idade entre 19 e $64 \operatorname{anos}(M=35,5 ; D P=10,24)$. Quanto à atividade ocupacional, $31,8 \%$ eram docentes, $28,41 \%$ atuavam em funções técnicas/assessorias, $19,3 \%$ eram profissionais liberais, $11,3 \%$ desempenhavam funções gerenciais e 9,2\% identificaram-se como estudantes/trabalhadores. O tempo médio de trabalho identificado foi de 14 anos $(D P=9,62)$ e de trabalho com TICs foi de 10,3 anos $(D P=7,12)$.

\section{Instrumentos}

Para atender aos objetivos do estudo foram utilizados os seguintes instrumentos autoaplicáveis:

1. Questionário sociodemográfico (sexo, idade, situação conjugal, filhos, escolaridade) e laboral (função, tempo de trabalho, tempo de trabalho com TICs, quantidade de horas de uso de TICs);

2. Dutch Work Addiction Scale (DUWAS) de Schaufeli, Taris e Bakker (2006) e adaptada para o uso no Brasil por Carlotto e Del Lí-bano (2010). A escala avalia a Adição ao Trabalho em duas dimensões: o Trabalho Compulsivo (cinco itens; $\alpha=0,70$ ) e o Trabalho Excessivo (cinco itens; $\alpha=0,74$ ). No total, constitui-se de dez itens avaliados por uma escala tipo Likert, variando de 1 (nunca) a 4 (todos os dias);

3. Escala de Tecnoestresse (RED/TIC) desenvolvida por Salanova, Llorens, Cifre e No- 
gareda (2004) e adaptada para o Brasil por Carlotto e Câmara (2010). A escala consiste de dezesseis questões, subdivididas em quatro dimensões: (a) Descrença - sentimento que o uso de TIC não traz benefícios ao seu trabalho (quatro itens; $\alpha=0,74$ ); (b) Fadiga - avalia o cansaço mental e cognitivo pelo uso continuado de TICs (quatro itens; $\alpha=0,89$ ); (c) Ansiedade - tensão frente ao uso de TICs (quatro itens; $\alpha=0,77$ ); e, (d) Ineficácia - sentimentos negativos sobre a própria capacidade e competência no uso de TICs (quatro itens; $\alpha=0,80$ ). Todos os itens são avaliados em escala tipo Likert de sete pontos, variando de 0 (nada/nunca) a 6 (sempre/todos os dias);

4. Escala de Satisfação com a Vida (ESV) de Diener, Emmons, Larsen e Griffin (1985), versão adaptada para o Brasil por Giacomoni e Hutz (1997). O instrumento composto por uma única dimensão (cinco itens; $\alpha=0,86$ ) que avalia o quanto as pessoas estão satisfeitas com sua vida por meio de uma escala tipo Likert de cinco pontos variando de 1 (discordo totalmente) a 7 (concordo totalmente).

\section{Procedimentos}

Os participantes foram recrutados pela técnica do Respondent Driven Sampling (RDS), na qual os primeiros participantes ( $1^{\mathrm{a}}$ onda) enviaram o convite para novos participantes ( $2^{\mathrm{a}}$ onda) até que se tenha atingido o tamanho desejado da amostra (Goel \& Salganik, 2009). Esta é uma técnica que permite o alcance de participantes com características semelhantes e que dispõem de tecnologia para acessar os instrumentos. Esse processo resulta em uma rede de recrutamento com características próprias que anulam possíveis vieses presentes na escolha inicial dos participantes (Goel \& Salganik, 2009). A amostragem baseada no RDS mostra-se útil porque produz uma amostra final independente daqueles que a iniciaram e traz informações de boa qualidade de forma rápida (Neiva-Silva, 2010).

O programa estatístico SPSS versão 17 (SPSS/PASW, Inc., Chicago, IL) foi utilizado para a análise de dados. Estatísticas descritivas foram realizadas para calcular as frequências, médias e desvios-padrão. A força e a direção das relações entre as variáveis foram determinadas usando-se a medida do coeficiente de correlação de Pearson. Antes de realizar a análise de regressão linear hierárquica (método Stepwise), foram verificados os pressupostos de multicolinearidade, normalidade, linearidade, homoscedasticidade e independência dos resíduos e outliers, sem que se identificasse violação que contraindicasse sua utilização. A análise assumiu como variável dependente as dimensões da adição ao trabalho, e como variáveis independentes as sociodemográficas, laborais, dimensões do tecnoestresse e satisfação com a vida. A seleção das variáveis preditoras assumiu o nível de significância de $p$ $<0,05$. Na análise de regressão, o poder do efeito foi obtido pelos coeficientes de regressão padronizados calculados para cada modelo final, de acordo com Field (2009).

\section{Considerações Éticas}

Foram respeitadas todas as orientações éticas, conforme Resolução 196 do Conselho Nacional de Saúde (Ministério da Saúde, 1997) no que diz respeito à pesquisa com seres humanos. O estudo possui aprovação pelo Comitê de Ética em Pesquisa da Universidade Luterana do Brasil, Protocolo n.2010/224H.

\section{Resultados}

Na Tabela 1 são apresentas as médias, os desvios-padrão, valores de alfa e matriz de correlação entre as variáveis em estudo. Os instrumentos revelam índices adequados de consistência interna avaliados pelo alfa de Cronbach, variando de 0,74 a 0,88 (Field, 2009). As variáveis apresentam correlações que se alteram de fracas $(r=0,21)$ a moderadas $(r=0,57)$. Os resultados indicam um poder de efeito alto $\left(R^{2}\right.$ $=0,257)$ e $\left(R^{2}=0,396\right)$ de acordo com orientação de Field (2009) que classifica como um alto poder de efeito valores obtidos entre 0,25 e 0,50 . 
Tabela 1

Estatísticas Descritivas, Alfas de Cronbach e Correlações entre as Variáveis em Estudo

\begin{tabular}{|c|c|c|c|c|c|c|c|c|c|c|c|c|c|c|c|}
\hline Variáveis & $M$ & $D P$ & $\alpha$ & (1) & (2) & (3) & (4) & (5) & (6) & (7) & (8) & (9) & (10) & (11) & (12) \\
\hline $\begin{array}{l}\text { 1. Trabalho } \\
\text { Excessivo }\end{array}$ & 2,57 & 0,69 & 0,81 & 1 & $0,54^{* *}$ & $-0,07$ & $0,57^{* *}$ & $0,46^{* *}$ & 0,20 & 0,09 & 0,12 & $-0,04$ & 0,09 & 0,05 & $-0,01$ \\
\hline $\begin{array}{l}\text { 2. Trabalho } \\
\text { Compulsivo }\end{array}$ & 2,15 & 0,65 & 0,77 & & 1 & 0,15 & $0,48^{* *}$ & $0,42^{* *}$ & $0,24^{*}$ & $-0,22^{*}$ & 0,16 & 0,04 & $-0,03$ & 0,01 & $-0,05$ \\
\hline 3. Descrença & 2,45 & 1,26 & 0,84 & & & 1 & 0,15 & $0,32^{* *}$ & $0,41^{* *}$ & $-0,16$ & $-0,17$ & 0,18 & $-0,14$ & 0,13 & 0,01 \\
\hline 4. Ansiedade & 2,60 & 1,43 & 0,88 & & & & 1 & $0,57^{* *}$ & $0,32^{* *}$ & $-0,12$ & $0,21^{*}$ & $-0,09$ & $-0,11$ & 0,06 & $-0,15$ \\
\hline 5. Fadiga & 1,88 & 1,33 & 0,74 & & & & & 1 & $0,64^{* *}$ & $-0,11$ & $-0,05$ & $-0,06$ & 0,05 & $-0,02$ & $-0,19$ \\
\hline 6. Ineficácia & 0,95 & 1,01 & 0,81 & & & & & & 1 & $-0,06$ & 0,00 & $0,25^{*}$ & $-0,04$ & 0,02 & $-0,08$ \\
\hline $\begin{array}{l}\text { 7. Satisfação } \\
\text { com a vida }\end{array}$ & 4,75 & 1,31 & 0,86 & & & & & & & 1 & 0,10 & $0,25^{*}$ & $-0,19$ & $0,31^{* *}$ & $0,22^{*}$ \\
\hline 8. Sexo ${ }^{a}$ & - & - & - & & & & & & & & 1 & 0,04 & $-0,18$ & 0,04 & $-0,19$ \\
\hline $\begin{array}{l}\text { 9. Idade } \\
\text { (anos) }\end{array}$ & 10,30 & 7,12 & - & & & & & & & & & 1 & $-0,26^{*}$ & 0,18 & $0,67^{* *}$ \\
\hline $\begin{array}{l}\text { 10. Estado } \\
\text { civil }\end{array}$ & - & - & - & & & & & & & & & & 1 & $-0,18$ & $-0,06$ \\
\hline $\begin{array}{l}11 . \\
\text { Escolaridade }\end{array}$ & 3,63 & 0,55 & & & & & & & & & & & & 1 & 0,16 \\
\hline $\begin{array}{l}\text { 12. Trabalho } \\
\text { c/ TICs } \\
\text { (anos) }\end{array}$ & 35,50 & 10,24 & - & & & & & & & & & & & & 1 \\
\hline
\end{tabular}

${ }^{\mathrm{a}} 0$ - Homem, 1 - Mulher.

$* p<0,05, * * p<0,01$.

Na Tabela 2, elencam-se os resultados das análises de regressão linear hierárquica para as duas dimensões da adição ao trabalho. Os resultados para a dimensão trabalho excessivo revelaram um modelo preditor composto por duas dimensões do tecnoestresse: ansiedade e descrença. O modelo explicou 39,6\% da variância dessa dimensão, assim, indicando que quanto maior a ansiedade $(\beta=0,624)$ e menor a descrença $(\beta=-0,229)$, maior é o trabalho excessivo.

No tocante à dimensão trabalho compulsivo, o modelo é explicado por uma única variável, a dimensão de ansiedade do tecnoestresse, que explicou $25,7 \%$ da sua variabilidade. Verifica-se que quanto maior a ansiedade $(\beta=0,507)$, maior é o trabalho compulsivo.

Tabela 2

Análise de Regressão Múltipla para as Dimensões de Adição ao Trabalho

\begin{tabular}{|c|c|c|c|c|c|c|c|}
\hline Variáveis & $R^{2}$ & $R^{2 \text { Ajustado }}$ & B & SE & $\beta$ & $t$ & $p$ \\
\hline \multicolumn{8}{|c|}{ Trabalho Excessivo } \\
\hline Ansiedade & 0,346 & 0,338 & 0,308 & 0,044 & 0,624 & 6,908 & $0,000^{*}$ \\
\hline Descrença & 0,396 & 0,381 & $-0,138$ & 0,055 & $-0,229$ & $-2,531$ & $0,013^{*}$ \\
\hline Modelo F & & & & $25,590^{*}$ & & & \\
\hline \multicolumn{8}{|c|}{ Trabalho Compulsivo } \\
\hline Ansiedade & 0,257 & 0,248 & 0,229 & 0,044 & 0,507 & 5,234 & $0,000^{*}$ \\
\hline Modelo F & & & & $27,396^{*}$ & & & \\
\hline
\end{tabular}




\section{Discussão}

O objetivo do presente estudo foi verificar se as variáveis sociodemográficas, laborais, tecnoestresse e satisfação com a vida prediziam a adição ao trabalho em trabalhadores que utilizam TICs. Os resultados obtidos confirmaram parcialmente a hipótese do estudo, revelando que somente as dimensões do tecnoestresse explicaram a adição ao trabalho, o que corrobora o modelo teórico utilizado por Salanova et al. (2013) e confirma a sobreposição destes conceitos.

Uma das possíveis explicações para este resultado e a relação entre os dois construtos pode se dever ao fato de que a inclusão das tecnologias da informação e comunicação como ferramenta fundamental de trabalho possibilitou o aumento do volume e carga horária de trabalho. Com a introdução da internet, de smartphones e tablets na vida cotidiana e no contexto de trabalho, a jornada de trabalho se prolongou para além do ambiente físico laboral, gerando um aumento importante de informações que circulam em alta velocidade fazendo com que aumente a pressão social para que sejam dadas respostas imediatas, fazendo com que todas as demandas existentes ganhem o caráter de urgência (Netto, Marcellino, \& Corrêa, 2010). É, hoje, possível realizar o trabalho em qualquer tempo e espaço físico que disponha de acesso de TICs. Os pressupostos de que o trabalhador é considerado um instrumento de trabalho e de consumo podem explicar os resultados. A manipulação e ideologização organizacionais fazem com que até mesmo o seu lazer se torne uma extensão do trabalho (Adorno \& Horkheimer, 1985).

Assim, cada vez mais, o trabalhador passa a depender das novas TICs, direta e/ou indiretamente, para executar suas atividades laborais, sendo-lhe exigidas capacitação e atualização constantes para se ajustar a estes novos recursos, assim como maior velocidade e dinamismo, em qualquer condição, como premissa organizacional. Estes fatos podem explicar comportamentos aditivos, ou seja, que o trabalhador desenvolva adição ao trabalho, podendo justificar, assim, o resultado encontrado no presente estudo. Como referido anteriormente, o trabalho permeado pelas TICs requer maior exigência cognitiva e, por esse motivo, maior é a sobrecarga em seus processos mentais. As crenças e estímulos internos (distorções cognitivas de onipotência, significado do trabalho, dentre outras) em interação com estímulos externos (exigências dos sistemas produtivos e pressão social), geram estresse e sofrimento psíquico (Ferreira, 2006). Diante das características da nova lógica de trabalho, competitiva e produtivista, é de se esperar que os níveis de ansiedade dos trabalhadores aumentem, assim como a crença de que as TICs são elementos fundamentais para o sucesso e sobrevivência no mercado laboral.

$\mathrm{Na}$ amostra investigada, verificou-se que a ansiedade foi a variável que apresentou maior poder explicativo para as duas dimensões da adição ao trabalho. A ansiedade frente ao uso de tecnologias é caracterizada como uma resposta afetiva (Simsek, 2011), sendo que esta emoção é também o componente central do estresse que pode conduzir à vivência de elevados níveis de ativação fisiológica e desconforto ao usar TICs (Salanova et al., 2013). O trabalhador pode sentir-se tenso ao interagir com computadores e outras ferramentas tecnológicas (Gaudron \& Vignoli, 2002; Maurer, 1983) em razão do grande volume de informações e atividades que pode e deve desenvolver. Além disso, as demandas e recursos tecnológicos são extremamente dinâmicos e, de certa forma, imprevisíveis, o que pode aumentar a ansiedade para dar conta das mesmas e, inclusive, provocar um estado de hipervigilância nestes trabalhadores. Estes elevados níveis de ansiedade podem ocasionar comportamentos de aumento de trabalho e do cumprimento de tarefas (visando um alívio de ansiedade) e podendo ocasionar, assim, a adição ao trabalho, explicação que corrobora os resultados encontrados.

Enquanto processo, em um primeiro momento, a ansiedade surge em função de ter de dar conta do trabalho excessivo, então, logo a seguir, pode se estabelecer o comportamento de trabalhar compulsivamente, dimensão central da adição ao trabalho (Douglas \& Morris, 2006; Salanova et al., 2008; Snir \& Harpaz, 2004). No caso da dimensão trabalho excessivo, verificou-se uma relação negativa da descrença; e positiva 
da ansiedade com esta dimensão. Tal fato pode sugerir que, além do aumento da ansiedade, acreditar que as TICs trazem benefícios profissionais ou são indispensáveis para as atividades laborais, aumenta o trabalho excessivo. Já a dimensão trabalho compulsivo foi explicada somente pela ansiedade. Pode-se pensar no conceito de tecnoadição, proposto por Salanova et al. (2013), que sugere que as pessoas sentem-se ansiosas quando não estão utilizando as TICs. Ainda, pode-se entender que as compulsões de diferentes ordens e naturezas estão sempre a serviço do alívio da ansiedade, o que não parece diferente no caso do trabalho compulsivo (American Psychiatric Association, 2002; Barlow \& Durand, 2011).

Os resultados encontrados contrariam estudos que identificaram que variáveis sociodemográficas, laborais (Carlotto, 2011; Schaufeli et al., 2006) e satisfação com a vida (Aziz \& Zickar, 2006; Bakker, Demeroti, \& Burke, 2009) estavam associadas à adição ao trabalho. Uma possível explicação para os resultados que não corroboram estudos anteriores diz respeito às particularidades da amostra investigada, considerando-se o contexto no qual se inserem estes trabalhadores e a interação dinâmica destas variáveis em determinado tempo sócio-histórico. Convém salientar que em um curto espaço de tempo, transformações expressivas em âmbito socioeconômico colaboraram para a expansão de inúmeros mercados, inclusive das TICs. O resultado deste estudo ajusta-se ao referido por Del Líbano, Rodriguez, Llorens, Cifre e Salanova (2006) que advertem que ainda não são conhecidas com exatidão as razões da ocorrência da adição ao trabalho. Talvez, deva-se à pressão das próprias organizações de trabalho, às circunstâncias particulares em que se encontram as pessoas, a características do indivíduo como fatores de personalidade ou, ainda, pode ser um conjunto de todos esses fatores.

Os resultados identificados merecem atenção, considerando que a adição ao trabalho pode ser explicada pelo tecnoestresse e ambos os processos, além de serem variáveis sobrepostas, atualmente são reforçados pelo contexto de trabalho e social. Ou seja, o cenário socioeconômico capitalista valoriza a produtividade e competitivi- dade. O objeto cuja dependência do indivíduo se estabelece - o trabalho - é atrativo, positivo e com influência socioambiental que, por sua vez, é capaz de reforçar esta dependência (Fatayer, 2008). O uso de TICs facilita o comportamento aditivo na medida em que proporciona maior agilidade e controle sobre o trabalho, aumentando a competividade típica do adicto ao trabalho. Segundo Salanova et al. (2008), estes profissionais apresentam alta vitalidade e competitividade e forte necessidade de controlar as tarefas e pessoas o tempo todo. São pessoas energéticas, motivadas e que apresentam alta centralidade e significado positivo atribuído ao trabalho. A satisfação obtida no trabalho e a avaliação de que o trabalho excessivo é executado por "uma boa causa" são, provavelmente, aspectos que reforçam o comportamento de trabalho excessivo. Há fatores adicionais, tais como a necessidade de realização e reconhecimento, no qual o desempenho é o maior indicador reconhecido pela organização (Baruch, 2011). Essas organizações, voltadas para o modo de gestão da excelência, priorizam o êxito, a adesão passional, a adaptabilidade e o constante desafio de forma à mobilização total do trabalhador a serviço da organização, dessa maneira, deixando-o vulnerável a patologias ocupacionais (Chanlat, 2000).

Assim, o elevado uso de TICs e o trabalho excessivo e compulsivo podem não ser questionados pelas organizações e trabalhadores. Pelo contrário, cada vez mais, indicadores quantitativos de produtividade/produção têm sido valorizados, e a competição no mercado acaba por naturalizar o trabalho fora do expediente, incluindo finais de semana e feriados, por exemplo. O entendimento do trabalho excessivo e da adição ao trabalho não é percebido como um problema de saúde, geralmente, quando o adoecimento do trabalhador ocorre e alguma ação, pessoal ou organizacional, é tomada quando observam-se comorbidades como a depressão, distúrbios do sono e alimentares e problemas psicossomáticos. Muitas vezes, o sofrimento e adoecimento ocorrem antes do trabalhador tornar-se um problema para a organização, uma vez que o trabalhador tende, de algum modo, a afastar-se de sua atividade, ou é afastado pela 
organização, com diagnósticos que, comumente, não refletem com precisão o que está de fato acontecendo (Borsoi, 2007).

O estudo apresenta algumas limitações a serem consideradas na leitura de seus resultados: a primeira é o seu delineamento transversal, o que impossibilita a análise de relações causais; a segunda a ser considerada é o tipo de amostra não probabilística, que inviabiliza a realização de generalizações. Ainda, como foram utilizadas apenas medidas de autorrelato, deve-se considerar a possibilidade de existência de algum tipo de viés em razão da desejabilidade social que algumas questões abordam quando o tema investigado é a sobrecarga e o estresse laboral (Razavi, 2001).

Os resultados obtidos podem fomentar futuras investigações, sobretudo as de delineamento longitudinal de modo a avaliar o efeito que a incorporação de TICs produz tanto nas políticas organizacionais como na qualidade de vida e saúde dos trabalhadores. Também pode ser relevante a inclusão de outras variáveis e medidas que possam aumentar o poder explicativo da adição ao trabalho, principalmente variáveis de personalidade, comprometimento com a carreira e desempenho.

Como implicações para a prática, pode-se pensar em ações preventivas em empresas, organizações e diferentes instituições com foco no planejamento de carreira, dimensionamento de metas e cobranças. Também, fazem-se importantes e necessárias intervenções direcionadas à psicoeducação sobre TICs - benefícios e malefícios - assim como no tocante à centralidade e significado atribuído ao trabalho pelos trabalhadores. Nesse sentido, faz-se necessária a ressignificação de crenças distorcidas relacionadas à produtividade, valor do trabalho e lazer, capacidade de delegar tarefas e perfeccionismo. Intervenções grupais podem ser igualmente eficazes, pois a adição ao trabalho provoca sérias dificuldades interpessoais e o reforçamento intragrupo pode ser benéfico (Wijhe et al., 2013).

Já não se pode pensar em um ambiente laboral sem utilização das TICs. Resta aos trabalhadores capacitarem-se e lidarem de forma saudável e adaptativa com estas ferramentas e formas de trabalho. Aos profissionais de saúde, cabe compreender a relação entre saúde/doença mental e trabalho, o reconhecimento dos determinantes das patologias ocupacionais ou do sofrimento psíquico e das efetivas mudanças que possam ser geradas pelos resultados encontrados neste campo (Borsoi, 2007).

A investigação acerca da adição ao trabalho, tecnoestresse e fatores associados torna a presente investigação uma contribuição importante para a ciência psicológica, uma vez que, somada ao crescimento paulatino dos trabalhadores que atuam com TICs, vem se observando uma série de consequências negativas para a saúde física e psíquica, ocasionadas pelo uso excessivo de tais ferramentas tecnológicas. Ao mesmo tempo, a elevada competitividade do mercado de trabalho e o foco na produção podem tornar difícil a identificação de casos de adição ao trabalho, a relação com tecnoestresse e, consequentemente, o desenvolvimento de intervenções voltadas a este fenômeno psicossocial. Acredita-se que, uma vez identificados os possíveis preditores da adição ao trabalho em trabalhadores que utilizam TICs, propostas interventivas e preventivas poderão ser melhor delineadas.

\section{Referências}

Adorno, T. W., \& Horkheimer, M. (1985). A dialética do esclarecimento. Rio de Janeiro, RJ: Jorge Zahar.

American Psychiatric Association. (2002). Manual diagnóstico e estatístico de transtornos mentais (4. ed. rev.). Porto Alegre, RS: Artmed.

Arla, D., Paquet, S., Scott, N., \& Hambley, L. (2012). Perceived information and communication technology (ICT) demands on employee outcomes: The moderating effect of organizational ICT support. Journal of Occupational Health Psychology, 17(4), 473-491. doi:10.1037/a0029837

Aziz, S., \& Zickar, M. J. (2006). A cluster analysis investigation of workaholism as a syndrome. Journal of Occupational Health Psychology, 11(1), 52-62. doi:10.1037/1076-8998.11.1.52

Bakker, A. B., Demerouti, E., \& Burke, R. (2009). Workaholism and relationship quality: A spillover- crossover perspective. Journal of Occupational Health Psychology, 14(1), 23-33. doi:10.1037/a0013290 
Barlow, D. H., \& Durand, V. M. (2011). Psicopatologia - Uma abordagem integrada. São Paulo, SP: Cengage Learning.

Baruch, Y. (2011). The positive wellbeing aspects of workaholism in cross cultural perspective: The chocoholism metaphor. Career Development International, 16(6), 572-591. doi:10.1108/13620431111178335

Beck, J. S. (1997). Terapia cognitiva: Teoria e prática. Porto Alegre, RS: Artmed.

Bonebright, C. A., Clay, D. L., \& Ankenmann, R. D. (2000). The relationship of workaholism with work-life conflict, life satisfaction, and purpose in life. Journal of Counseling Psychology, 47(4), 469-477. doi:10.1037/0022-0167.47.4.469

Borsoi, I. C. F. (2007). Da relação entre trabalho e saúde à relação entre trabalho e saúde mental. Psicologia \& Sociedade, 19, 103-111. doi:10.1590/S0102-71822007000400014

Carlotto, M. S. (2011). Adição ao trabalho e relação com fatores de risco sociodemográficos, laborais e psicossociais. Psico-USF, 16(1), 87-95. doi:10.1590/S1413-82712011000100010

Carlotto, M. S., \& Câmara, S. G. (2010). Tradução, adaptação e exploração de propriedades psicométricas da Escala de Tecnoestresse. Psicologia em Estudo, 15(1), 157-164.

Carlotto, M., \& Del Líbano (2010). Tradução, adaptação e exploração de propriedades psicométricas da Escala de Adição ao Trabalho "Dutch Work Addiction Scale" (DUWAS). Contextos Clínicos, 3(2), 141-150.

Chanlat, J.-F. (2000). Modos de gestão, saúde e segurança no trabalho. In E. Davel \& J. M. Vasconcelos (Eds.), Recursos humanos e subjetividade (pp. 118-128). Petrópolis, RJ: Vozes.

Del Líbano, M., Rodríguez, A. M., Llorens, S., Cifre, E., \& Salanova, M. (2006). Adicción al trabajo y Flow: una relación conflictiva. Riesgo Laboral, 14, 34-37.

Diener, E., Emmons, R. A., Larsen, R., \& Griffin, S. (1985). The Satisfaction with Life Scale. Journal of Personality Assessment, 49, 91-95. doi:10.1207/s15327752jpa4901_13

Douglas, E. J., \& Morris, R. J. (2006). Workaholic, or just hard worker? Career Development International, 11, 394-417. doi:10.1108/13620430610683043
Elowe, J. (2010). Workaholism: Between illusion and addiction. L'Encéphale, 36(4), 285-293. doi:10.1016/j.encep.2009.12.002

Fatayer, J. (2008). Addiction types: A clinical sociology perspective. Journal of Applied Social Science, 2, 88-93. doi:10.1177/193672440800200107

Ferreira, A. P. C. (2006). Tecnologia de Informação, controle e mundo do trabalho: Pensar tecnologia na ótica do trabalhador. Revista Eletrônica de Ciências Sociais, 11, 14-24.

Field, A. (2009). Discovering statistics with SPSS $\left(2^{\text {nd }}\right.$ ed.). London: Sage.

Gaudron, J. P., \& Vignoli, E. (2002). Assessing computer anxiety with the interaction model of anxiety: Development and validation of the computer anxiety trait subscale. Computers in Human Behavior, 18, 315-325. doi:10.1016\%2 FS0747-5632\%2801\%2900039-5 (xds:string)

Giacomoni, C. H., \& Hutz, C. S. (1997). A mensuração do bem-estar subjetivo: Escala de Afeto Positivo e Negativo e Escala de Satisfação de Vida [Resumo]. In Sociedade Interamericana de Psicologia (Ed.), Anais do XXVI Congresso da Sociedade Interamericana de Psicologia (p. 313). São Paulo, SP: Sociedade Interamericana de Psicologia.

Goel, S., \& Salganik, M. J. (2009). Respondent-driven sampling as Markov chain Monte Carlo. Statistics in Medicine. 28, 2202-2229. doi:10.1002/ $\operatorname{sim} .3613$

Instituto Brasileiro de Geografia e Estatística. (2013). O setor de tecnologia da informação e comunicação no Brasil (2003-2006). Recuperado em 14 de julho de 2013, de http://www.ibge.gov.br/ home/estatistica/economia/stic/publicacao.pdf

Killinger, B. (1991). Workaholic: The respectable addicts. New York: Simon \& Schuster.

Maurer, M. M. (1983). Development and validation of a measure of computer anxiety (Unpublished master's thesis). Iowa State University, Ames, USA.

Ministério da Saúde. (1997). Diretrizes e normas para pesquisa envolvendo seres humanos. Resolução CNS 196/196. Brasília, DF: Autor.

Moreno-Jiménez, B., Gálvez-Herrer, M., GarrosaHernández, E., \& Rodríguez Carvajal, R. (2005). La adiccion al trabajo. Psicología Conductual, 13(3), 417-428. 
Neiva-Silva, L. (2010). Estudo comportamental com crianças e adolescentes em situação de rua em Porto Alegre e Rio Grande: Uso da técnica de Respondent Driven Sampling (RDS) para identificação de comportamentos sexuais de risco e uso de drogas - Relatório Final de Pesquisa (Relatório). Porto Alegre, RS: Universidade Federal do Rio Grande do Sul.

Netto, A. F. de N., Marcellino, N. C., \& Corrêa, D. A. (2010). Trabalho, tecnologia e lazer na sociedade contemporânea. Impulso, 20(50), 73-84.

Pacheco, W., Ferreira, C., Jr., Pereira, V. L. V., \& Pereira, H. V., Filho. (2005). A era da tecnologia da informação e da comunicação e a saúde do trabalhador. Revista Brasileira de Medicina do Trabalho, 3(2), 114-122.

Pocinho, M. D., \& Garcia, J. C. (2008). Impacto psicosocial de la tecnología de información y comunicación (TIC): tecnoestrés, daños físicos y satisfacción laboral. Acta Colombiana de Psicología, 11(2), 127-139.

Porter, G., \& Kakabadse, N. K. (2006). HRM perspective on addiction to technology and work. Journal of Management Development, 25, 535560. doi:10.1108/02621710610670119

Razavi, T. (2001). Self-report measures: An overview of concerns and limitations of questionnaire use in occupational stress research (Discussion Paper in Accounting and Management Science No. 01-175). Southampton, UK: School of Management, University of Southampton. Retrieved from http://eprints.soton.ac.uk/35712/

Salanova, M., Del Libano, M., Llorens, S., Schaufeli, W. B., \& Fidalgo, M. (2008). La adicción al trabajo (Nota Técnica de Prevención, 759, 22. Serie). Madrid, España: Instituto Nacional de Seguridad e Higiene en el Trabajo.

Salanova, M., Llorens, S., \& Cifre, E. (2013). The dark side of technologies: Technostress among users of information and communication technologies. International Journal of Psychology, 48(3), 422-436. doi:10.1080/00207594.2012.68 0460

Salanova, M., Llorens, S., Cifre, E., \& Nogareda, C. (2004). Tecnoestrés: concepto, medida e intervención psicosocial (Nota Técnica de Prevención). Barcelona, España: Centro Nacional de Condiciones de Trabajo.
Salanova, M., Llorens, S., Cifre, E., \& Nogareda, C. (2007). El tecnoestrés: concepto, medida e intervención psicosocial (Nota Técnica de Prevención, 730, 21. Serie). Madrid, España: Instituto Nacional de Seguridad e Higiene en el Trabajo.

Schaufeli, W. B., Taris, T. W.m \& Bakker, A. (2006). Dr. Jekyll and Mr. Hide: On the differences between work engagement and workaholism. In R. J. Burke (Ed.), Research companion to working time and work addiction (pp. 193-217). Northampton, MA: Edward Elgar.

Schiffrin, H., Edelman, A., Falkenster, M., \& Steward, C. (2010). The associations among computer mediated communication, relationships, and wellbeing. CyberPsychology, Behavior, and Social Networking, 13, 299-306. doi:10.1089/ cyber.2009.0173

Serva, M., \& Ferreira, J. L. (2006). O fenômeno workaholic na gestão de empresas. RAP, 40(2), 179-200.

Simsek, A. (2011). The relationship between computer anxiety and computer self-efficacy. Contemporary Educational Technology, 2(3), 177-187.

Snir, R., \& Harpaz, I. (2004). Attitudinal and demographic antecedents of workaholism. Journal of Organizational Change Management, 17(5), 520-536. doi:10.1108/09534810410554524

Taris, T. W., \& Schaufeli, W. B. (2007). Workaholism. In W. B. Schaufeli \& A. B. Bakker (Eds.), De psychologie van arbeid en gezondheid (pp. 359-372). Houten, Netherlands: Bohn Stafleu vanLoghum.

Wijhe, C. V., Peeters, M., \& Schaufeli, W. (2013). Irrational beliefs at work and their implications for workaholism. Journal of Occupational Rehabilitation, 23(3), 336-346. doi:10.1007/ s10926-012-9416-7 